\title{
The inositol regulon controls viability in Candida glabrata
}

\begin{abstract}
Correspondence
Todd B. Reynolds

treynol6@utk.edu
\end{abstract}

Received 16 April 2009

Revised 8 October 2009

Accepted 27 October 2009

\author{
Emily K. Bethea, Billy J. Carver, Anthony E. Montedonico \\ and Todd B. Reynolds
}

Department of Microbiology, University of Tennessee, Knoxville, TN 37996, USA

\section{INTRODUCTION}

Fungi of the genus Candida are the most common cause of human fungal infections and can lead to both mucosal and systemic infections (Calderone, 2002). Candida albicans is the most common cause of these infections, but nonalbicans Candida species are increasingly associated with disease (Coleman et al., 1998). One of these species, Candida glabrata, is now the second most common cause of both mucosal and systemic Candida infections (Kaur et al., 2005).

Phylogenetically, C. glabrata is more closely related to the non-pathogenic yeast Saccharomyces cerevisiae than most of the other common species of Candida associated with human disease (Kaur et al., 2005). C. glabrata lacks a number of the virulence factors associated with Candida pathogens, such as secreted hydrolases and hyphal growth (Kaur et al., 2005). Despite this, C. glabrata is a growing challenge in clinical settings where it causes mucosal infections and is associated with approximately $15 \%$ of all Candida-related systemic bloodstream infections (Pfaller \& Diekema, 2004). These observations are interesting in light of the fact that C. glabrata is more closely related to $S$.

Abbreviations: 5-FOA, 5-fluororotic acid; NCR, non-coding region, UAS ${ }_{\text {INO }}$, upstream activator sequence for inositol regulation. cerevisiae, but is still a pathogen, whereas $S$. cerevisiae is only very rarely associated with infection (Piarroux et al., 1999). A clearer understanding of how S. cerevisiae and $C$. glabrata differ from one another may help shed light on why one is a significant human pathogen and the other is not.

A number of recent studies have shown that C. albicans is transcriptionally rewired compared to $S$. cerevisiae. For example, the genes encoding enzymes of the Leloir pathway for galactose catabolism in S. cerevisiae are regulated by the ScGal4p transcription factor. However, in C. albicans these genes are regulated by the transcription factor Cphlp, while the C. albicans CaGal4p homologue regulates TCA cycle genes such as CaLAT1 (Martchenko et al., 2007). Other examples of transcriptional rewiring between $C$. albicans and $S$. cerevisiae include regulatory systems controlling mating type (Tsong et al., 2003), mitochondrial ribosomal genes (Ihmels et al., 2005) and de novo myoinositol biosynthesis genes (Hoppen et al., 2007; Y. L. Chen \& T. B. Reynolds, unpublished). Myo-inositol will be referred to as inositol throughout the rest of this article.

Since there are several examples of transcriptional rewiring between these two more distantly related yeasts ( $S$. cerevisiae and C. albicans), it was of interest to determine if similar rewiring is present between the more closely 
related yeasts $C$. glabrata and S. cerevisiae. The Leloir enzymes for galactose metabolism are not present in $C$. glabrata (Kaur et al., 2005), and this yeast is a galactose auxotroph (Kreger-vav Rij, 1984), so this pathway is unavailable for comparison. Mating has never been described for C. glabrata, thus this pathway is not useful for study either. In contrast to these pathways, the inositol regulon appears to be an excellent pathway to compare between these two yeasts. The inositol regulon is a very well-studied transcriptional regulon in $S$. cerevisiae (reviewed by Chen et al., 2007; Greenberg \& Lopes, 1996), and there are C. glabrata orthologues for both the transcription factors and targets of the S. cerevisiae inositol regulon (see Results and Discussion).

The inositol regulon in S. cerevisiae has been well-described (Chen et al., 2007; Greenberg \& Lopes, 1996), and consists of three main transcription factors that regulate target gene expression in response to extracellular inositol levels (Fig. 1). The roles of each of these transcription factors in controlling this regulon are described in more detail below. Transcriptional targets of the inositol regulon include a number of phospholipid biosynthetic genes, but the most highly expressed and well-characterized of these targets is the S. cerevisiae INO1 (ScINO1) gene. ScINO1 encodes an enzyme that occupies the rate-limiting step in de novo inositol biosynthesis. Inositol is essential and is required for the synthesis of phosphatidylinositol (PI) which is a precursor for several essential lipids, including inositol-phosphate signalling lipids, glycosylphosphatidylinositol (GPI) anchors and sphingolipids (Dickson \& Lester, 1999; Michell, 2008; Strahl \& Thorner, 2007).

ScInolp is an inositol-3-phosphate synthase which converts glucose 6-phosphate to inositol 3-phosphate

(a)
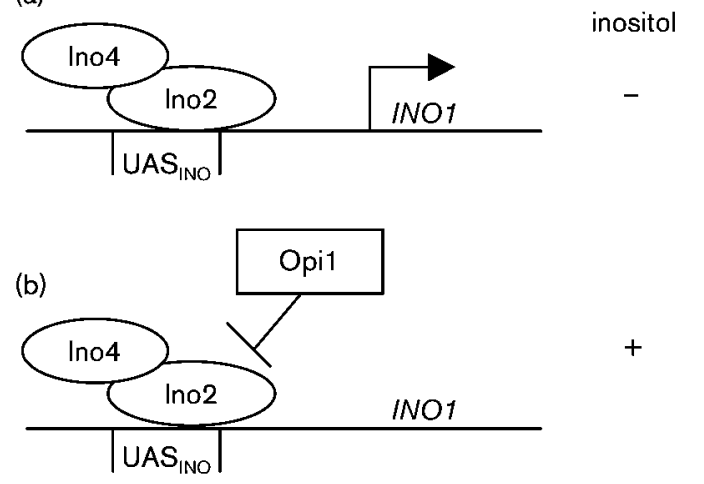

(Majumder et al., 1997). Inositol 3-phosphate is dephosphorylated by the inositol monophosphatases ScInm1p or ScInm2p to create inositol (Lopez et al., 1999). An Scino1s mutant cannot make inositol de novo and is an inositol auxotroph. In the absence of extracellular inositol (or at low concentrations like $10 \mu \mathrm{M})$ ScINO1 is expressed, and in the presence of higher concentrations of extracellular inositol ScINO1 is repressed (Graves \& Henry, 2000) (Fig. 1).

The inositol regulon transcription factors ScIno2p and ScIno4p form a heterodimeric transcriptional activator that binds to the upstream activator sequence $\left(\mathrm{UAS}_{\mathrm{INO}}\right)$ in the promoters of target genes such as ScINO1 (Ambroziak \& Henry, 1994; Bachhawat et al., 1995; Loewen et al., 2003; Lopes \& Henry, 1991; Nikoloff \& Henry, 1994; Schwank et al., 1995). Both ScIno2p and ScIno4p are absolutely required for transcription of ScINO1, so Scino2A and Scino $4 \Delta$ mutants are inositol auxotrophs.

The regulation of ScINO1 in response to extracellular inositol is dependent on the repressor protein ScOpilp (Heyken et al., 2005; Jiranek et al., 1998; Loewen et al., 2003, 2004; Loewen \& Levine, 2005). ScOpilp senses the level of extracellular inositol indirectly based on the level of the PI precursor lipid phosphatidic acid (PA). Inositol is the rate-limiting metabolite in PI synthesis, and when there is abundant extracellular inositol, PI synthesis is maximal, and PA levels are lower because PA is consumed during synthesis of PI. In these conditions ScOpilp binds to ScIno2p and represses transcription of ScINO1. When extracellular inositol is not available (or greatly decreased), PI synthesis slows, and PA levels increase in the endoplasmic reticulum (ER). ScOpilp, which binds to $\mathrm{PA}$, is recruited to the ER where it also binds Scs $2 \mathrm{p}$. Thus, the ScIno2p-ScIno4p heterodimer is freed to transcribe ScINO1. A mutation that deletes ScOPI1 results in constitutive overexpression of ScINO1 and other genes carrying the $\mathrm{UAS}_{\mathrm{INO}}$ sequence in their promoters.

The purpose of this study was to determine if C. glabrata carried an inositol regulon that is similar to that in $S$. cerevisiae, or if these yeasts are transcriptionally rewired for inositol regulation. In order to do this, C. glabrata homologues of the $S$. cerevisiae inositol regulon proteins were identified and disrupted. Analysis of these mutants revealed the surprising finding that CgOPI1 is essential. CgIno2p and CgIno4p (which are not essential) are activators of CgINO1, and CgOpilp is a transcriptional repressor of CgINO1. This is similar to the inositol regulon in S. cerevisiae. However, unlike in S. cerevisiae, CgOPI1 is required for viability. This difference from $S$. cerevisiae may indicate that the inositol regulon in C. glabrata has some additional or different targets than in bakers' yeast, or C. glabrata may be metabolically rewired compared to $S$. cerevisiae.

\section{METHODS}

Strains and media. Strains are listed in Table 1. Integrations and manipulations were confirmed by PCR (Table 2) in most cases and by 
Table 1. Strains used in this study

\begin{tabular}{|c|c|c|}
\hline Strain & Genotype & Reference \\
\hline BG14 & Cgura3:: Tn903neoR & Cormack \& Falkow (1999) \\
\hline BG2 & CgURA3, parent of BG14 & Cormack \& Falkow (1999) \\
\hline Cg11 & Cgura3:: Tn903neoR pCgOPI1 & This study \\
\hline $\operatorname{Cg} 12$ & Cgura3:: Tn903neoR pGRB2.1 & This study \\
\hline Cg14 & Cgura3:: Tn903neoR Cgopila pCgOPI1 & This study \\
\hline $\mathrm{Cg} 5$ & Cgura3:: Tn903neoR Cgino2s & This study \\
\hline $\mathrm{EBCg} 019$ & Cgura3:: Tn903neoR Cgino2s:: CgINO2 & This study \\
\hline $\operatorname{Cg} 23$ & Cgura3:: Tn903neoR Cgino2s pCgOPI1 & This study \\
\hline $\operatorname{Cg} 18$ & Cgura3:: Tn903neoR Cgino2s Cgopi1s pCgOPI1 & This study \\
\hline $\mathrm{EBCg} 005$ & Cgura3:: Tn903neoR Cgino4s & This study \\
\hline EBCg008 & Cgura3:: Tn903neoR Cgino4s:: CgINO4 & This study \\
\hline EBCg014 & Cgura3:: Tn903neoR Cgino1s & This study \\
\hline EBCg017 & Cgura3:: Tn903neoR Cgino1s:: CgINO1 & This study \\
\hline $\operatorname{Cg} 33$ & Cgura3:: Tn903neoR Cghis3s & This study \\
\hline EBCg046 & Cgura3:: Tn903neoR Cghis3s Cgtrp1s & This study \\
\hline EBCg048 & Cgura3::Tn903neoR Cghis3s Cgtrp1s pINTG4 pEB48 & This study \\
\hline EBCg049 & Cgura3:: Tn903neoR Cghis3s Cgtrp1s pINTG4 pEB48 & This study \\
\hline
\end{tabular}

Southern blotting with the tetO::HOP1::CgOPI1 strains. Strain BG14, which is a uracil auxotroph (Table 1), was a gift from Brendan Cormack and was used to generate all of the strains reported in this study. Disruption of CgINO2, CgINO4 and CgINO1 was performed in BG14 (Table 1) utilizing a two-step gene disruption strategy (Cormack \& Falkow, 1999; Cormack et al., 1999; Rothstein, 1991) with disruption constructs built in the pRS306 vector (Sikorski \& Hieter, 1989; Table 3) carrying the ScURA3 marker that can be selected for on media lacking uracil and counterselected against using media containing 5-fluororotic acid (5-FOA). Reconstitution of gene disruption mutants was done by transforming the strains with ScURA3-marked integrating plasmids carrying the wild-type gene (Table 3).

The Cgopils mutant (Cg14, Table 1) was generated in BG14 by transforming it with the pCgOPI1 episomal plasmid (ScURA3 marker, Table 3), and then disrupting the CgOPIl gene on the chromosome with the Cgopil:: NAT1 construct amplified from plasmid pCgopil $\triangle$ (Table 3). The Cgopi1s Cgino2 $\Delta$ double mutant was made in the same manner as the Cgopils mutant except it was done in strain $\operatorname{Cg} 5($ Cgino2 $\Delta)$.

The tetO:: HOP1:: CgOPI1 strains EBCg048 and EBCg049 (Table 1) were generated by first making BG14 a histidine and tryptophan auxotroph (Cghis3 $\operatorname{Cgtrp1\Delta }$ ). This was done by disrupting CgHIS3 in BG14 as described above for the Cgino2A gene to create strain Cg33. The CgTRP1 gene was disrupted in Cg33 using a plasmid from Karl Kuchler's lab to create strain EBCg046. EBCg046 was used to create the tetO:: HOPI1:: CgOPI1 strains EBCg048 and EBCg049 by first integrating plasmid pINTG4 carrying the tetR::GAL4AD repressor-activator into its genome as described by Nakayama et al. (1998). Then the tetO::HOP1::CgOPI1 construct was PCR-amplified from plasmid pEB48 (Table 3) and was used to replace the CgOPI1 locus on the chromosome.

Media used in this study included $2 \%$ agar plates or liquid media made with YPD, YNB, or inositol-free media (Styles, 2002) that included supplements of amino acids, nucleotides, inositol, doxycycline, 5-FOA, etc., as described in the text. For inositol-free agar plates, Bactoagar was used because it does not contain trace amounts of inositol.

Plasmids and constructs. The gene disruption plasmids (pRS306ino2 $\Delta$, pRS306-ino4 $\Delta$ and pRS306-ino1 $\Delta$ ) were made by PCR- amplifying DNA corresponding to approximately $500 \mathrm{bp}$ of noncoding DNA that flanked the $5^{\prime}$ and $3^{\prime}$ edges of each ORF $\left(5^{\prime}\right.$ or $3^{\prime}$ NCRs), and then cloning them into pRS306 adjacent to one another to create a disrupted allele (primers and restriction sites used for cloning are listed in Table 2). The pRS306-his3 $\Delta$ disruption plasmid was created in a similar manner by subcloning the CgHIS3 5 ' NCR into plasmid pGRB2.1 (Frieman et al., 2002) upstream of the $3^{\prime}$ NCR of CgHIS3 contained in this plasmid. The whole $5^{\prime}$ and $3^{\prime}$ NCR disruption cassette was then subcloned into the pRS306 integrating vector (Sikorski \& Hieter, 1989) as an $\mathrm{XbaI-KpnI} \mathrm{fragment} \mathrm{to} \mathrm{create}$ pRS306-his3 $\Delta$. The disrupted alleles from all of the pRS306 disruption cassette plasmids were used to replace the respective wild-type alleles on the chromosome of BG14 by the two-step deletion strategy (Cormack \& Falkow, 1999; Cormack et al., 1999; Rothstein, 1991). For example, the pRS306-ino1 $\Delta$ cassette was cut with $N r u$ I in the $5^{\prime}$ NCR to linearize it and target it to recombine upstream of CgINO1. PCR was used to confirm correct integrations and disruptions. In a similar manner, plasmids pRS306-ino4 $\Delta$, pRS306-ino1 $\Delta$ and pRS306-his3 $\Delta$ were cut with PmlI, SnaBI and $B g l I I$, respectively. Following replacement of the wild-type genes with the disrupted alleles, the mutant alleles were confirmed based on phenotype and PCR. Reintegration constructs were generated for each of the above disruptants in the vector pRS306 by PCR-amplifying the corresponding ORFs plus $\sim 500$ bp $5^{\prime}$ and $3^{\prime}$ NCRs with the primers $\mathrm{BCO} 3$ and $\mathrm{BCO} 2$ for $\mathrm{CgINO} 2$, USO1 and USO4 for CgINO4, and $\mathrm{MHO} 3$ and $\mathrm{MHO} 2$ for $\mathrm{CgINO} 1$. Cut sites for subcloning are listed in Table 2. Plasmids were linearized with the enzymes mentioned above, and transformed into their respective disruptant strains. Correct integrations were confirmed by PCR with primers listed in Table 2.

The episomal plasmid pCgOPI1 carrying CgOPI1 plus 215 bp of upstream DNA and 439 bp of downstream DNA was generated by amplifying the CgOPI1 ORF and flanking sequences from purified BG14 DNA using primers TRO605 and TRO608, and cutting the PCR product with BamHI and SpeI enzymes. The SpeI site was introduced by primer TRO608, and the BamHI site was internal to the amplified DNA fragment. This PCR product was cloned into the ScURA3bearing C. glabrata CEN/ARS plasmid pGRB2.1 (Frieman et al., 2002) using SpeI and BamHI. Plasmid pCgopils was generated by subcloning the NAT1 cassette from pAG25 (Goldstein \& McCusker, 1999) with primers TRO652 and TRO653 into an EcoRI site between a 469 bp fragment containing the $5^{\prime}$ NCR of CgOPI1 (cloned by 
Table 2. Primers used in this study

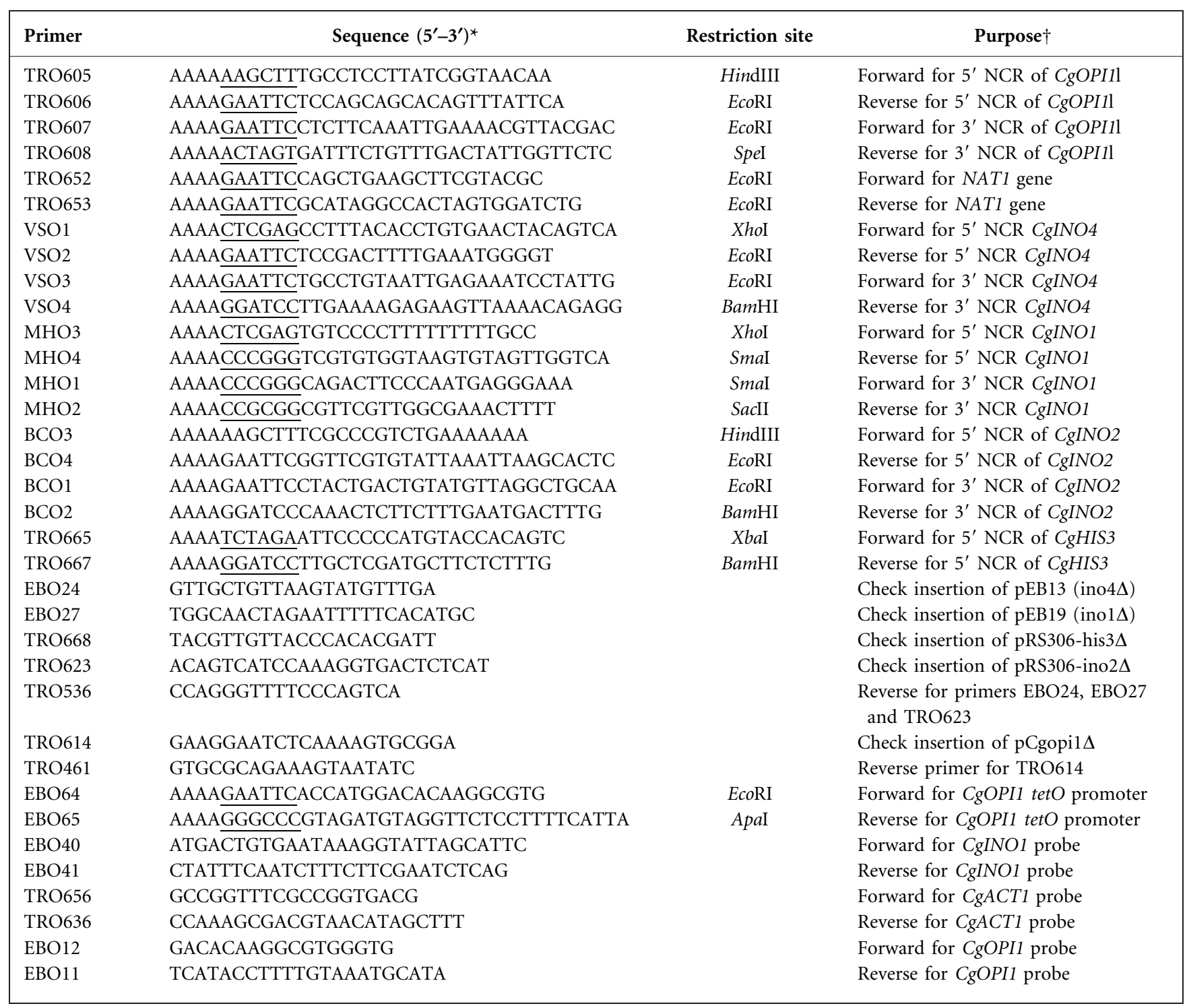

${ }^{\star}$ Underline shows the restriction site.

$\dagger \mathrm{NCR}$, Non-coding region that is either $5^{\prime}$ or $3^{\prime}$ to the designated ORF.

primers TRO605 and TRO606) and a $439 \mathrm{bp}$ fragment containing the 3' NCR of CgOPI1 (cloned by primers TRO607 and TRO608), both carried on the pRS306 vector. The pINT4 vector was a gift from Hironobu Nakayama (Nakayama et al., 1998) and was integrated into the EBCg046 genome after cutting it with EcoRV (Nakayama et al., 1998). Plasmid pEB48 was generated by subcloning an $\sim 500$ bp $5^{\prime}$ NCR of the CgOPIl ORF into p97CGH upstream $\left(5^{\prime}\right)$ of the HIS3 tetOHOP1 cassette using primers EBO76 and EBO77. The CgOPI1 ORF was then subcloned $3^{\prime}$ to the HIS3 tetO-HOP1 cassette using a PCR product made by primers EBO64 and EBO65. This whole segment from pEB48, containing 5'-CgOPI1, NCR-HIS3 and the tetO-HOP1-CgOPI1 ORF was then transformed into strain EBCg046 carrying pINTG4 to replace the $C g O P I 1$ promoter with the tetO-HOP1 cassette.

Northern blotting. Northern blotting was performed essentially as described by Reynolds (2006) using probes generated from primers EBO40 and EBO41 for CgINO1, EBO11 and EBO12 for CgOPI1,
TRO656 and TRO636 for CgACT1, TRO480 and TRO648 for ScACT1, EBO56 and EBO57 for CgCHO1, EBO54 and EBO55 for $\mathrm{CgOPI} 3$, and $\mathrm{EBO} 58$ and $\mathrm{EBO} 59$ for $\mathrm{CgCHO}$. Probes specific for ScACT1 or CgACT1 were used to reprobe Northerns for CgACT1 expression in C. glabrata for normalization, and both probes appeared to work well.

\section{RESULTS}

\section{CgINO2 and CgINO4 encode transcriptional activators of the CgINO1 gene and control de novo inositol biosynthesis}

A homologue of ScINO1 was identified in C. glabrata by BLAST analysis of the ScINO1 translated protein sequence 
Table 3. Plasmids used in this study

\begin{tabular}{|lllcl|}
\hline Plasmid & \multicolumn{1}{c}{ Description } & \multicolumn{1}{c|}{ Type } & Restriction site & Reference \\
\hline pRS306-ino2 $\Delta$ & CgINO2 knockout & Integrating & SnaBI & This study \\
pRS306-CgINO2 & CgINO2 reintegration & Integrating & SnaBI & This study \\
pEB19 & CgINO1 knockout & Integrating & NruI & This study \\
pEB21 & CgINO1 reintegration & Integrating & NruI & This study \\
pEB13 & CgINO4 knockout & Integrating & PmlI & This study \\
pEB17 & CgINO4 reintegration & Integrating & PmlI & This study \\
pCgOPI1 & Contains CgOPI1 & Episomal & This study \\
pCgopi1 & Cgopi1 $:$ NAT1 & PCR template & & This study \\
pRS306-his3 & CgHIS3 knockout & Integrating & BglII & This study \\
pGRB2.1 & ScURA3 vector & Episomal & Frieman et al. (2002) \\
pAG25 & NAT1 cassette & PCR template & Goldstein \& McCusker (1999) \\
pEB48 & tetO::HOP1::CgOPI1 & Integrating & This study \\
pINTG4 & tetR::GAL4AD & Integrating & & Nakayama et al. (1998) \\
pRS306 & Shuttle vector & Integrating & & Sikorski \& Hieter (1989) \\
\hline
\end{tabular}

against the C. glabrata genome at the Genolevures website (www.genolevures.org). The BLAST search revealed that the gene CAGL0I06050g encoded a protein that was $73.9 \%$ identical to ScInolp. We refer to CAGLOI06050g as CgINO1 based on the experiments described below.

CgINO1 expression was examined in media containing or lacking inositol by Northern blotting which revealed that CgINO1 was highly expressed in medium lacking inositol, but it was poorly expressed in medium containing $75 \mu \mathrm{M}$ inositol (Fig. 2). This is similar to what has been observed for ScINO1 in S. cerevisiae (Graves \& Henry, 2000). In order to determine if $\mathrm{CgINO1}$ was required for de novo inositol biosynthesis, the CgINO1 ORF was disrupted by homologous recombination using a two-step gene deletion strategy (Cormack \& Falkow, 1999; Cormack et al., 1999). The resulting Cgino1s mutant was unable to grow on inositol-free medium (Fig. 3). However, when the CgINO1 gene was reintegrated into the genome at the CgINO1 locus, the reconstituted strain (Cgino1 $1:$ :CgINO1) could grow in medium lacking inositol (Fig. 3).

The CgINO1 gene is regulated in a similar manner as the ScINO1 gene in synthetic medium containing or lacking inositol, which suggests that the inositol regulon that controls ScINO1 in S. cerevisiae might be conserved in C. glabrata. In order to test this, homologues of the ScINO2 and ScINO4 transcriptional activator genes were identified by BLAST searches, querying the protein sequences of ScIno2p and ScIno4p against the C. glabrata genome at the Genolevures website. The BLAST searches revealed only one strong homologue for each protein, and these were encoded by the genes CAGLOB01947g for ScIno2p (35.6\% identity) and CAGLOI07359g for ScIno4p (44.5\% identity). These genes, CAGLOB01947g and CAGLOI07359g, were named $\mathrm{CgINO} 2$ and $\mathrm{CgINO}$, respectively. CgINO2 and CgINO4 were disrupted using the two-step gene disruption method which completely removed the ORF of each gene. The resulting Cgino $2 \Delta$ and Cgino $4 \Delta$ mutants were tested to determine if they could grow in the absence of inositol in the medium. As seen for orthologous Saccharomyces mutants, the Cgino2s and Cgino4s mutants were unable to grow on inositol-free medium (Fig. 3). These data suggest that $\mathrm{CgINO} 2$ and $\mathrm{CgINO} 4$ control the expression of the CgINO1 gene. Northern blotting revealed that Cgino2 $\Delta$ and Cgino $4 \Delta$ mutants showed a complete lack of CgINO1 expression even in inositol-free medium (Fig. 2). Reconstituted Cgino2s::CgINO2 and Cgino4s::CgINO4 strains, conversely, grew well on medium lacking inositol (Fig. 3) and regulated CgINO1 much like the wild-type strain (Fig. 2).

\section{CgOPI1 is an essential gene in C. glabrata}

In $S$. cerevisiae, the $S c O P I 1$ gene encodes the main regulator of de novo inositol biosynthesis, and its homologue was identified in a BLAST search against the C. glabrata genome at Genolevures as described above. The protein encoded by CAGLOK03267/g was found to be $52.3 \%$ identical to ScOpilp. An attempt was made to disrupt the CgOPIl gene by the two-step gene disruption strategy; however, this method continuously yielded strains carrying a wild-type ORF of CgOPI1.

The above results suggested that $C g O P I 1$ might be essential, and this was shown to be the case using a counterselectable episomal plasmid expressing CgOPI1. CgOPI1 was cloned, along with non-coding DNA flanking both $5^{\prime}$ and $3^{\prime}$ of the ORF (including the transcriptional promoter and terminator, respectively), into the CEN/ARS vector pGRB2.1 (Frieman et al., 2002) to create plasmid pCgOPI1. Plasmid pCgOPI1, which carries the S. cerevisiae URA3 gene, was transformed into the wild-type strain, and the chromosomal CgOPI1 ORF was disrupted by homologous recombination using a construct in which the CgOPI1 ORF is replaced with the nourseothricin resistance marker cassette NAT1 (Goldstein \& McCusker, 1999). The resulting pCgOPI1 Cgopi1s strain was then streaked onto medium containing 5-FOA. Processing of 5-FOA by the 
(a)

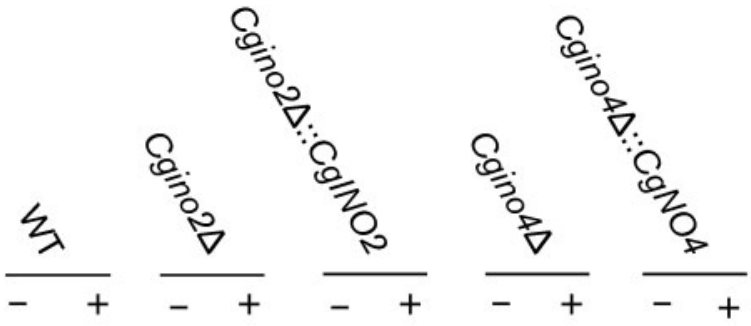

INO1

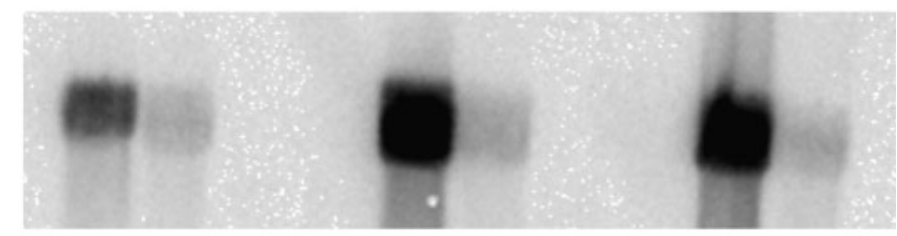

ACT1

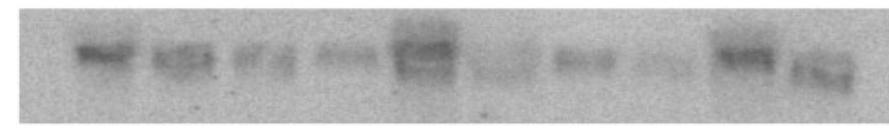

(b)

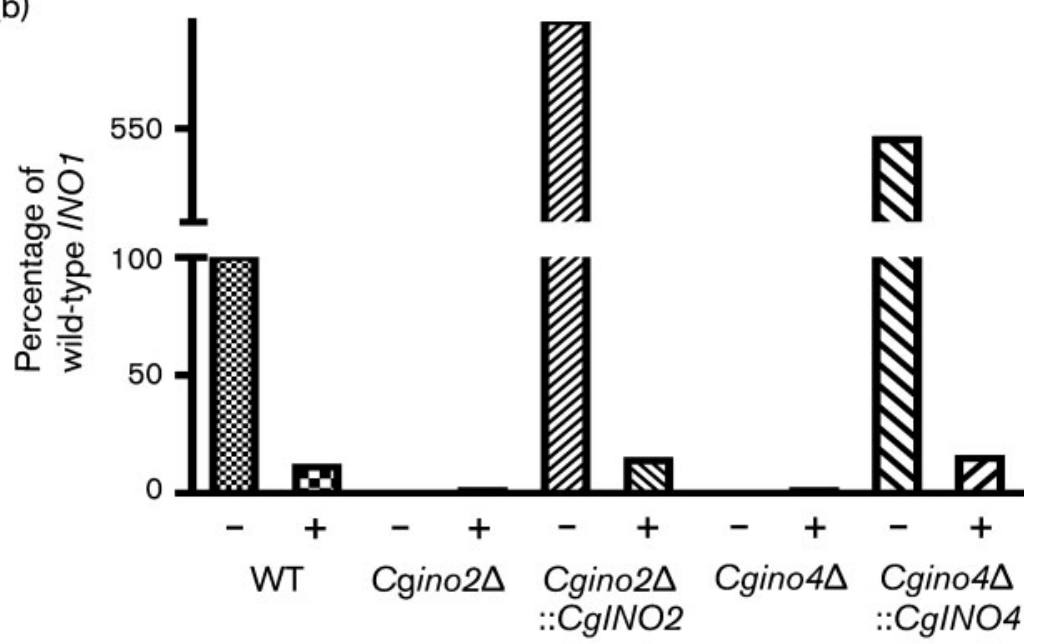

Fig. 2. CgINO2 and CgINO4 are required to express CgINO1 in the absence of exogenous inositol. (a) Northern blotting was used to assess the expression of CgINO1 in wild-type (WT), mutant and reconstituted strains in the presence $(+)$ or absence $(-)$ of exogenous inositol. CgACT1 was used as a loading control. Strains were grown overnight at $30{ }^{\circ} \mathrm{C}$ in inositol-free medium supplemented with $75 \mu \mathrm{M}$ inositol, washed with water, and resuspended in inositol-free media containing either 0 or $75 \mu \mathrm{M}$ inositol, after which the cultures were incubated with shaking for $6 \mathrm{~h}$ before collecting total RNA for Northern blotting. (b) A Storm Phosphorimager was used to quantify expression of $\mathrm{CgINO} 1$ in each strain relative to the wild-type strain grown in inositol-free medium, which was arbitrarily set at $100 \%$. Expression was normalized to CgACT1 expression. These results are representative of four different experiments.
URA3 gene product from S. cerevisiae or C. glabrata leads to production of 5-fluorouracil which is toxic to $C$. glabrata (Boeke et al., 1987; Cormack \& Falkow, 1999).

If the Cgopils mutation is lethal, then no Cgopils pCgOPI1 colonies should grow on 5-FOA medium because the 5-FOA would select against the cells carrying pCgOPI1. In Fig. 4 it is clear that the Cgopild pCgOPI1 strain cannot grow on 5-FOA. In contrast, colonies from the parental strain carrying pCgOPI1 or the empty vector grew well on this medium, indicating that they had lost the plasmid but were still viable.

These experiments were performed in the BG2 strain background (Cormack \& Falkow, 1999). To be sure that this phenotype was not strain-specific, the above strategy was used to test the essentiality of CgOPI1 in the ATCC 2001 strain background (a gift from Karl Kuchler). It was found that CgOPI1 was essential in strain ATCC 2001 as well, which suggested that the essentiality of $C g O P I 1$ is not just a BG2 strain-specific phenomenon (data not shown).

\section{The regulation of viability by $\mathrm{CgOPI1}$ is dependent on the CgINO2 transcription factor}

Based on the model from S. cerevisiae (Fig. 1), it was hypothesized that disruption of CgOPI1 causes overexpression of a downstream target of the inositol regulon that then results in a loss of viability. In $S$. cerevisiae, overexpression of ScINO1 in the Scopila mutant is due to unrepressed transcriptional activation by ScIno2p (Fig. 1). The ScINO1 overexpression phenotype of the Scopils mutant can be blocked by a Scino $2 \Delta$ mutation. The Scopil $\Delta$ Scino $2 \Delta$ double mutant acts like the Scino2 $\Delta$ single mutant and fails to express $S c I N O 1$ because the Scino2 $\Delta$ mutation is epistatic to the Scopi1s mutation (Graves \& Henry, 2000).

If the Cgopils mutant compromises viability due to overexpression of a downstream target of CgIno2p, then 

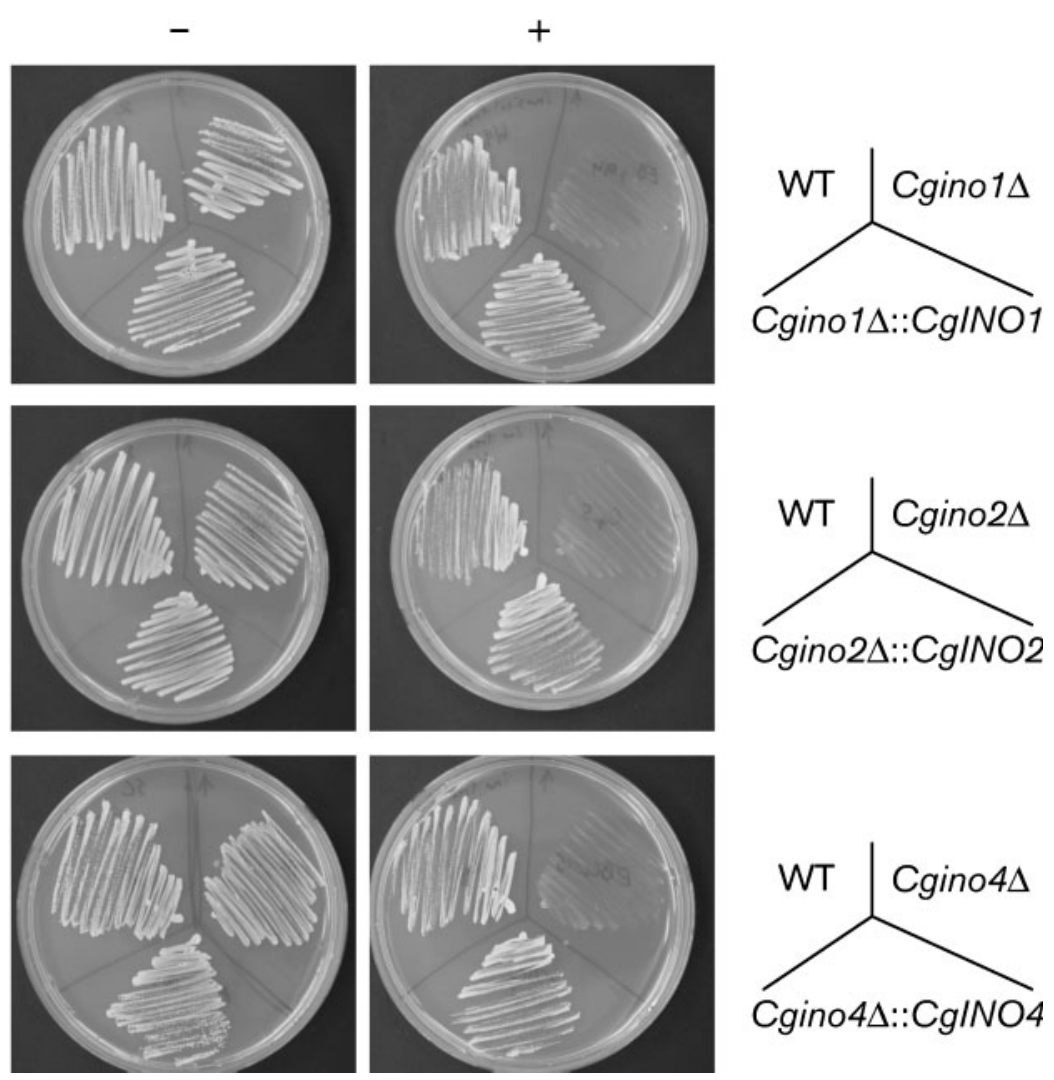

Fig. 3. The CgINO1, CgINO2 and CgINO4 genes are all required for de novo inositol biosynthesis, like their $S$. cerevisiae homolo-

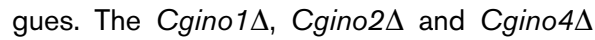
mutants and their respective reconstituted strains (along with the wild-type control) were streaked onto inositol-free media supplemented with $0(-)$ or $75 \mu \mathrm{M}$ inositol $(+)$, and grown at $30{ }^{\circ} \mathrm{C}$ for 3 days. a Cgino2s mutation should restore the viability of a Cgopils mutant by blocking expression of this putative target. In order to test this hypothesis, the Cgino2 $\Delta$ mutant was transformed with plasmid pCgOPI1, and the chromosomal ORF of CgOPI1 was disrupted as described

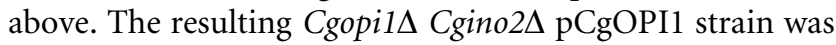
streaked onto 5-FOA medium, and it was found to grow like the wild-type strain carrying $\mathrm{pCgOPI} 1$ or empty vector (Fig. 5). The Cgino2s strain also grew like the wild-type strain (Fig. 5).

\section{The CgOPI1 gene product represses expression of CgINO1}

The results above suggest that CgOpilp can act as a repressor of CgIno2p targets such as CgINO1. To determine if CgOpilp could repress CgINO1, CgOPI1 was placed under the control of a doxycycline-repressible promoter (Nakayama et al., 1998). C. glabrata strain BG14 (Cgura3s) was modified by disrupting both the CgHIS3 gene (making it a histidine auxotroph) and the CgTRP1 gene (making it a tryptophan auxotroph). Using the resulting triple auxotroph (Cgura3s Cghis3s Cgtrp1s), the promoter of the CgOPI1 gene was replaced on the chromosome by homologous recombination with the tetO-HOP1 promoter construct derived from plasmid p97CGH (CgHIS3) (Nakayama et al., 1998). This strain was further modified by integration of plasmid pINTG4 (CgTRP1) carrying the tetR:: GAL4AD-encoded repressoractivator that activates the tetO-HOP 1 chimeric promoter in the absence of doxycycline, but represses it in the presence of doxycycline.
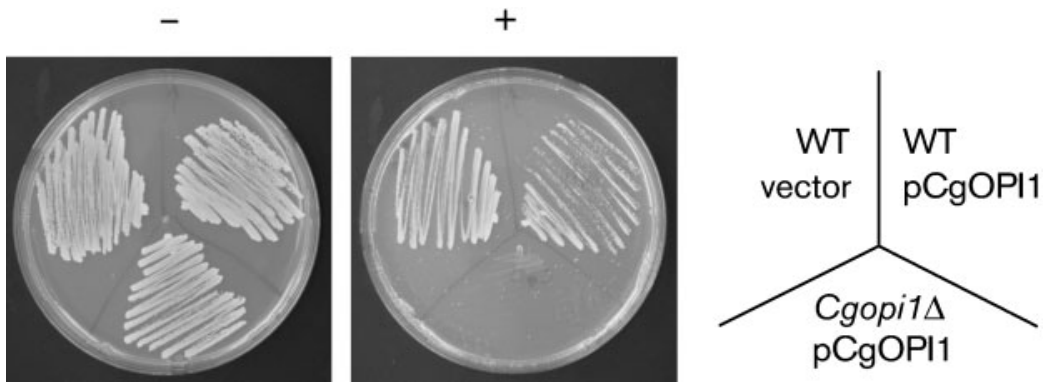

Fig. 4. The $\mathrm{CgOP} / 1$ gene is essential. Wildtype (WT) and Cgopi1s strains carrying CgOPI1 on a URA3 plasmid (pCgOPI1) were grown for 3 days on medium with $(+)$ or without (-) 5-FOA at $30{ }^{\circ} \mathrm{C}$. 

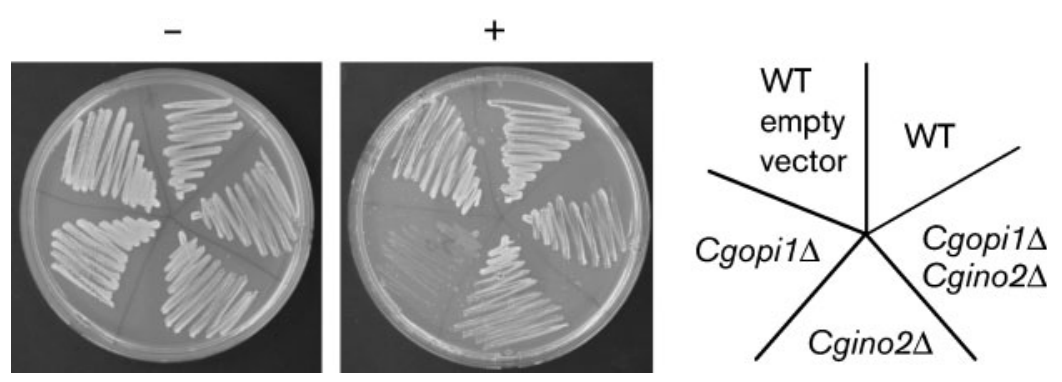

Fig. 5. The viability defect of the Cgopi1 mutant is dependent on the CgINO2 transcriptional activator. The wild-type (WT), Cgopi1s, Cgino2s and Cgopi1s Cgino2s strains containing plasmid pCgOPI1 (URA3based) were streaked onto synthetic medium plates with (+) or without (-) 5-FOA. The WT strain containing the empty vector was included as a control.

The resulting strain was then tested for growth in the presence and absence of doxycycline, and it was found that this strain showed very poor growth in $10 \mu \mathrm{g}$ doxycycline $\mathrm{ml}^{-1}$, but grew quite well in the absence of doxycycline (Fig. 6b). These experiments were performed on synthetic minimal medium, but similar results were seen in YPD (rich) medium (E. K. Bethea \& T. B. Reynolds, unpublished). When CgOPI1 levels were assessed in this strain by Northern blotting, it was found that $C g O P I 1$ was expressed in the absence of doxycycline, but was not expressed in the presence of the drug (Fig. 6a).

CgOpilp is a repressor of CgINO1. When CgOPI1 is repressed by doxycycline, CgINO1 expression increases substantially, but when $C g O P I 1$ is expressed in the absence of doxycycline, then CgINO1 mRNA is very low or undetectable (Fig. 6a). This indicates that CgOpilp acts as a repressor of CgINO1. We were also able to demonstrate the repression of CgINO1 expression by CgOpilp using the C. glabrata copper-inducible MTII promoter (El Barkani et al., 2000) as well (E. K. Bethea \& T. B. Reynolds, unpublished).

\section{Overexpression of $\mathrm{CgINO1}$ is not responsible for the loss of viability in the Cgopi1 $\Delta$ mutant}

Since CgINO1 is overexpressed in the absence of CgOPI1, it seemed possible that $C g I N O 1$ overexpression is responsible for the loss of viability. This was tested by creating a Cgopils Cgino1s pCgOPIl double mutant. However, when this double mutant is streaked onto 5-FOA it fails

(a)

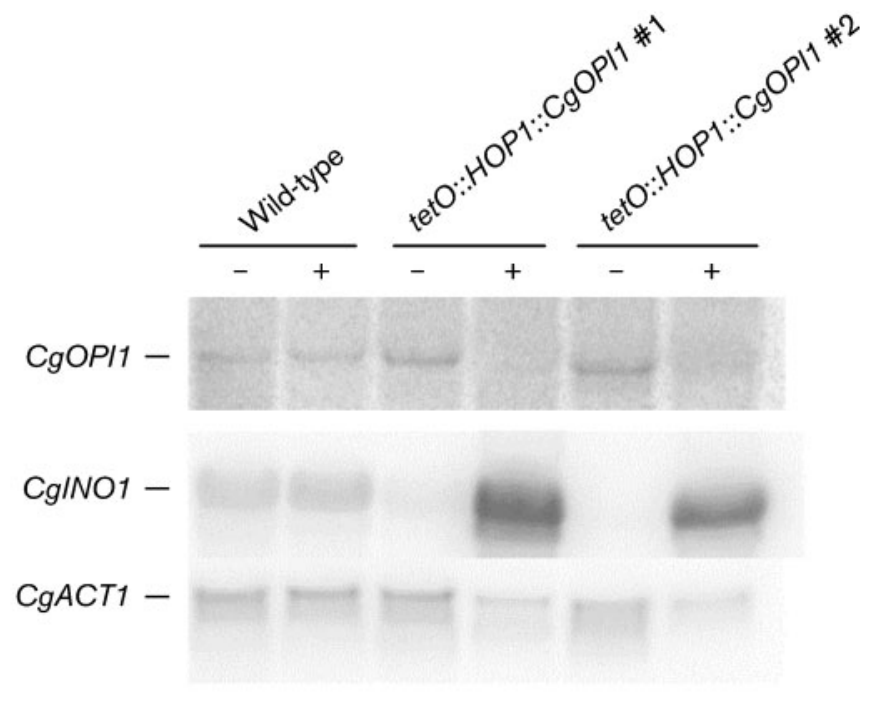

(b)

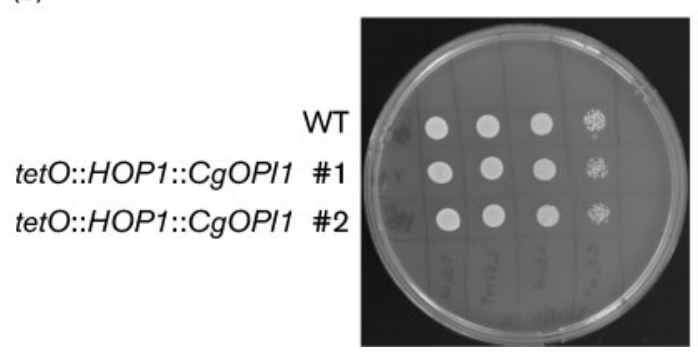

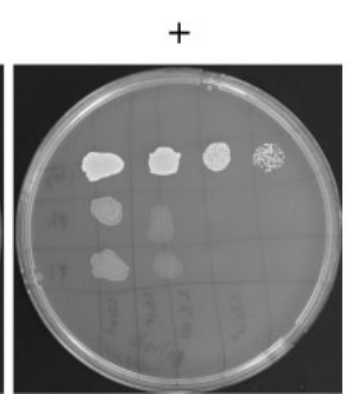

Fig. 6. CgOpi1p is a transcriptional repressor of CgINO1. The promoter of the chromosomal copy of $\mathrm{CgOP} / 1$ was replaced with the doxycycline-repressible tetO::HOP1 promoter. (a) Expression of $\mathrm{CgOP} / 1, \mathrm{Cg} / \mathrm{NO} 1$ and $\mathrm{CgACT1}$ (loading control) were tested by Northern blotting in the presence $(+)$ and absence (-) of doxycycline. Cells were grown to saturation overnight in synthetic medium (containing $\sim 11 \mu \mathrm{M}$ inositol) lacking doxycycline, washed with water, and were then resuspended in fresh synthetic medium containing 0 or $10 \mu \mathrm{g}$ doxycycline $\mathrm{ml}^{-1}$ and grown for $\sim 6 \mathrm{~h}$ at $30^{\circ} \mathrm{C}$ with shaking at which time samples were taken for Northern blotting. (b) Cells were grown on plates containing $0(-)$ or $10 \mu \mathrm{g}$ doxycycline $\mathrm{ml}^{-1}(+)$ to confirm that loss of $\mathrm{CgOP} / 1$ expression decreased viability. Two different transformants were tested for the doxycycline repressible promoter. 
to grow (E. K. Bethea \& T. B. Reynolds, unpublished) indicating that the disruption of CgINO1 is not sufficient to restore viability as seen with $\mathrm{CgINO} 2$ (Fig. 5).

\section{DISCUSSION}

Transcriptional rewiring appears to exist between the transcription factor homologues of the inositol regulons of S. cerevisiae and C. albicans (Hoppen et al., 2007; Y. L. Chen \& T. B. Reynolds, unpublished). C. glabrata is much more closely related to $S$. cerevisiae than $C$. albicans based on phylogenetic trees comparing $18 \mathrm{~S}$ ribosomal sequences (Kaur et al., 2005). Our analysis suggests that the $C$. glabrata inositol regulon is not transcriptionally rewired compared to S. cerevisiae, at least for CgINO1 regulation. However, a major difference between the two species is that the OPI1 homologue in C. glabrata appears to be essential (Figs 4-6), whereas it is not in S. cerevisiae.

It is not clear why the Cgopil $\Delta$ mutation is lethal, but our data suggest that the Cgopi1 $\Delta$ mutation causes overexpression of CgIno2p-CgIno4p target genes and one of these targets causes a loss in viability when overexpressed. In support of this hypothesis, disruption of CgINO2 in the Cgopils strain rescues growth of the Cgopil $\Delta$ mutant, presumably because the downstream target is no longer overexpressed (Fig. 5).

One possible target for compromising viability appeared to be CgINO1. However, disruption of CgINO1 in the Cgopils strain did not rescue viability on 5-FOA plates (E. K. Bethea \& T. B. Reynolds, unpublished). This indicates that CgINO1 overexpression is not toxic.

There are two main models to explain the loss of viability of the Cgopi1 $\Delta$ mutant. (1) A direct downstream target gene involved in phospholipid biosynthesis is overexpressed, and C. glabrata is particularly sensitive to this imbalance in lipid biosynthesis and loses viability. (2) Expression of a direct target gene not involved in lipid biosynthesis is affected by Cgopils and results in a loss of viability.

In the first model, there are several phospholipid biosynthetic genes that may be targets of the C. glabrata inositol regulon based on sequence similarity to homologues in S. cerevisiae. Direct downstream targets of the $S$. cerevisiae inositol regulon have been identified by the presence of the UAS INO consensus sequence CATGTGAAAT in their promoters and their misregulation

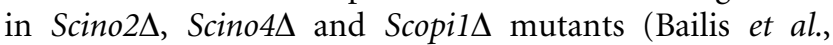
1992; Graves \& Henry, 2000; Jackson \& Lopes, 1996; Lai \& McGraw, 1994; Li \& Brendel, 1993). Using these genes as a guide, there are five genes in C. glabrata that contain the sequence CATGTG (the most important part of the UAS $_{\text {INO }}$ consensus sequence; Greenberg \& Lopes, 1996) in their promoters. These genes include CgINO1, CgOPI3, $\mathrm{CgCHO} 1, \mathrm{CgCHO} 2$ and CgITR2. In addition to these five genes, two other genes, CgERG20 and CgCKI1, contain the sequence CATGTT, which differs by only one base and could possibly also respond to the inositol regulon.
In the second model, the target may be unrelated to phospholipid biosynthesis and/or may not have a homologue that is regulated by the inositol regulon in $S$. cerevisiae. Such a gene might also not be the direct cause of the loss of viability, but might itself regulate a downstream effector and cause the loss of viability. For example, if CgOPI1 were to repress a transcriptional repressor of an essential gene, then loss of $\mathrm{CgOPI1}$ could result in loss of expression of the essential gene and compromise viability.

The essential nature of Opilp in C. glabrata appears to be unique among the few characterized members of the growing Opilp family of proteins, a family that is specific to fungi (Hirakawa et al., 2009). Opilp homologues in S. cerevisiae, C. albicans, and Yarrowia lipolytica (Yas3p) are not essential (Hirakawa et al., 2009; Jiranek et al., 1998) (Y. L. Chen \& T. B. Reynolds, unpublished). It is of interest to note that in S. cerevisiae and C. glabrata, two closely related yeasts, the Opilp proteins both regulate inositol biosynthesis, whereas in the more distantly related yeasts $C$. albicans and $Y$. lipolytica, their Opilp family members, CaOpilp (C. albicans) and Yas3p (Y. lipolytica) do not regulate inositol biosynthesis (Endoh-Yamagami et al., 2007; Hirakawa et al., 2009; Yamagami et al., 2004) (Y. L. Chen \& T. B. Reynolds, unpublished). Thus, the Opilp family of proteins may have a number of diverse functions.

We are currently investigating the mechanism by which CgOPI1 controls viability in C. glabrata. Considering that the Opilp family of proteins is unique to fungi, $\mathrm{CgOpilp}$ may be a possible future drug target for treating C. glabrata infections.

\section{ACKNOWLEDGEMENTS}

We gratefully acknowledge Brendan Cormack for supplying us with strains, plasmids, and a great deal of advice without which this work would not have been possible. We also are grateful to Karl Kuchler, Tobias Schwarzmueller and Helmut Jungwirth for sending us strains and plasmids. We are grateful to Hironobu Nakayama for sending us the doxycycline-repressible plasmid system, and Fritz Muhlschlegel for the MTII promoter plasmids. This work was supported by grants AHA 0765366B and NIH-1R03AI071863-01A1.

\section{REFERENCES}

Ambroziak, J. \& Henry, S. A. (1994). INO2 and INO4 gene products, positive regulators of phospholipid biosynthesis in Saccharomyces cerevisiae, form a complex that binds to the INO1 promoter. J Biol Chem 269, 15344-15349.

Bachhawat, N., Ouyang, Q. \& Henry, S. A. (1995). Functional characterization of an inositol-sensitive upstream activation sequence in yeast. A cis-regulatory element responsible for inositol-choline mediated regulation of phospholipid biosynthesis. J Biol Chem 270, 25087-25095.

Bailis, A. M., Lopes, J. M., Kohlwein, S. D. \& Henry, S. A. (1992). Cis and trans regulatory elements required for regulation of the $\mathrm{CHO} 1$ gene of Saccharomyces cerevisiae. Nucleic Acids Res 20, 1411-1418.

Boeke, J. D., Trueheart, J., Natsoulis, G. \& Fink, G. R. (1987). 5 -Fluoroorotic acid as a selective agent in yeast molecular genetics. Methods Enzymol 154, 164-175. 
Calderone, R. A. (2002). Candida and Candidiasis. Washington, DC: American Society for Microbiology.

Chen, M., Hancock, L. C. \& Lopes, J. M. (2007). Transcriptional regulation of yeast phospholipid biosynthetic genes. Biochim Biophys Acta 1771, 310-321.

Coleman, D. C., Rinaldi, M. G., Haynes, K. A., Rex, J. H., Summerbell, R. C., Anaissie, E. J., Li, A. \& Sullivan, D. J. (1998). Importance of Candida species other than Candida albicans as opportunistic pathogens. Med Mycol 36 (Suppl. 1), 156-165.

Cormack, B. P. \& Falkow, S. (1999). Efficient homologous and illegitimate recombination in the opportunistic yeast pathogen Candida glabrata. Genetics 151, 979-987.

Cormack, B. P., Ghori, N. \& Falkow, S. (1999). An adhesin of the yeast pathogen Candida glabrata mediating adherence to human epithelial cells. Science 285, 578-582.

Dickson, R. C. \& Lester, R. L. (1999). Yeast sphingolipids. Biochim Biophys Acta 1426, 347-357.

El Barkani, A., Haynes, K., Mosch, H., Frosch, M. \& Muhlschlegel, F. A. (2000). Candida glabrata shuttle vectors suitable for translational fusions to lac $Z$ and use of beta-galactosidase as a reporter of gene expression. Gene 246, 151-155.

Endoh-Yamagami, S., Hirakawa, K., Morioka, D., Fukuda, R. \& Ohta, A. (2007). Basic helix-loop-helix transcription factor heterocomplex of Yas1p and Yas2p regulates cytochrome P450 expression in response to alkanes in the yeast Yarrowia lipolytica. Eukaryot Cell 6, 734-743.

Frieman, M. B., McCaffery, J. M. \& Cormack, B. P. (2002). Modular domain structure in the Candida glabrata adhesin Epalp, a betal,6 glucan-cross-linked cell wall protein. Mol Microbiol 46, 479-492.

Goldstein, A. L. \& McCusker, J. H. (1999). Three new dominant drug resistance cassettes for gene disruption in Saccharomyces cerevisiae. Yeast 15, 1541-1553.

Graves, J. A. \& Henry, S. A. (2000). Regulation of the yeast INO1 gene. The products of the INO2, INO4 and OPI1 regulatory genes are not required for repression in response to inositol. Genetics 154, 1485-1495.

Greenberg, M. L. \& Lopes, J. M. (1996). Genetic regulation of phospholipid biosynthesis in Saccharomyces cerevisiae. Microbiol Rev 60, $1-20$.

Heyken, W. T., Repenning, A., Kumme, J. \& Schuller, H. J. (2005). Constitutive expression of yeast phospholipid biosynthetic genes by variants of Ino2 activator defective for interaction with Opil repressor. Mol Microbiol 56, 696-707.

Hirakawa, K., Kobayashi, S., Inoue, T., Endoh-Yamagami, S., Fukuda, R. \& Ohta, A. (2009). Yas3p, an Opil-family transcription factor regulates cytochrome $\mathrm{P} 450$ expression in response to n-alkanes in Yarrowia lipolytica. J Biol Chem 284, 7126-7137.

Hoppen, J., Dietz, M., Warsow, G., Rohde, R. \& Schuller, H. J. (2007). Ribosomal protein genes in the yeast Candida albicans may be activated by a heterodimeric transcription factor related to Ino2 and Ino4 from S. cerevisiae. Mol Genet Genomics 278, 317-330.

Ihmels, J., Bergmann, S., Gerami-Nejad, M., Yanai, I., McClellan, M., Berman, J. \& Barkai, N. (2005). Rewiring of the yeast transcriptional network through the evolution of motif usage. Science 309, 938-940.

Jackson, J. C. \& Lopes, J. M. (1996). The yeast UME6 gene is required for both negative and positive transcriptional regulation of phospholipid biosynthetic gene expression. Nucleic Acids Res 24, 1322-1329.

Jiranek, V., Graves, J. A. \& Henry, S. A. (1998). Pleiotropic effects of the opil regulatory mutation of yeast: its effects on growth and on phospholipid and inositol metabolism. Microbiology 144, 2739-2748.

Kaur, R., Domergue, R., Zupancic, M. L. \& Cormack, B. P. (2005). A yeast by any other name: Candida glabrata and its interaction with the host. Curr Opin Microbiol 8, 378-384.
Kreger-vav Rij, N. J. W. (1984). The Yeasts - a Taxonomic Study, 3rd edn. Amsterdam: Elsevier.

Lai, K. \& McGraw, P. (1994). Dual control of inositol transport in Saccharomyces cerevisiae by irreversible inactivation of permease and regulation of permease synthesis by INO2, INO4, and OPI1. J Biol Chem 269, 2245-2251.

Li, Z. \& Brendel, M. (1993). Co-regulation with genes of phospholipid biosynthesis of the CTR/HNM1-encoded choline/nitrogen mustard permease in Saccharomyces cerevisiae. Mol Gen Genet 241, 680-684.

Loewen, C. J. \& Levine, T. P. (2005). A highly conserved binding site in vesicle-associated membrane protein-associated protein (VAP) for the FFAT motif of lipid-binding proteins. J Biol Chem 280, 1409714104 .

Loewen, C. J., Roy, A. \& Levine, T. P. (2003). A conserved ER targeting motif in three families of lipid binding proteins and in Opilp binds VAP. EMBO J 22, 2025-2035.

Loewen, C. J., Gaspar, M. L., Jesch, S. A., Delon, C., Ktistakis, N. T., Henry, S. A. \& Levine, T. P. (2004). Phospholipid metabolism regulated by a transcription factor sensing phosphatidic acid. Science 304, 1644-1647.

Lopes, J. M. \& Henry, S. A. (1991). Interaction of trans and cis regulatory elements in the INO1 promoter of Saccharomyces cerevisiae. Nucleic Acids Res 19, 3987-3994.

Lopez, F., Leube, M., Gil-Mascarell, R., Navarro-Avino, J. P. \& Serrano, R. (1999). The yeast inositol monophosphatase is a lithiumand sodium-sensitive enzyme encoded by a non-essential gene pair. Mol Microbiol 31, 1255-1264.

Majumder, A. L., Johnson, M. D. \& Henry, S. A. (1997). 1L-myoinositol-1-phosphate synthase. Biochim Biophys Acta 1348, 245-256.

Martchenko, M., Levitin, A., Hogues, H., Nantel, A. \& Whiteway, M. (2007). Transcriptional rewiring of fungal galactose-metabolism circuitry. Curr Biol 17, 1007-1013.

Michell, R. H. (2008). Inositol derivatives: evolution and functions. Nat Rev Mol Cell Biol 9, 151-161.

Nakayama, H., Izuta, M., Nagahashi, S., Sihta, E. Y., Sato, Y., Yamazaki, T., Arisawa, M. \& Kitada, K. (1998). A controllable geneexpression system for the pathogenic fungus Candida glabrata. Microbiology 144, 2407-2415.

Nikoloff, D. M. \& Henry, S. A. (1994). Functional characterization of the INO2 gene of Saccharomyces cerevisiae. A positive regulator of phospholipid biosynthesis. J Biol Chem 269, 7402-7411.

Pfaller, M. A. \& Diekema, D. J. (2004). Twelve years of fluconazole in clinical practice: global trends in species distribution and fluconazole susceptibility of bloodstream isolates of Candida. Clin Microbiol Infect 10 (Suppl. 1), 11-23.

Piarroux, R., Millon, L., Bardonnet, K., Vagner, O. \& Koenig, H. (1999). Are live saccharomyces yeasts harmful to patients? Lancet 353 , 1851-1852.

Reynolds, T. B. (2006). The Opilp transcription factor affects expression of FLO11, Mat formation, and invasive growth in Saccharomyces cerevisiae. Eukaryot Cell 5, 1266-1275.

Rothstein, R. (1991). Targeting, disruption, replacement, and allele rescue: integrative DNA transformation in yeast. Methods Enzymol 194, 281-301.

Schwank, S., Ebbert, R., Rautenstrauss, K., Schweizer, E. \& Schuller, H. J. (1995). Yeast transcriptional activator INO2 interacts as an Ino2p/Ino4p basic helix-loop-helix heteromeric complex with the inositol/choline-responsive element necessary for expression of phospholipid biosynthetic genes in Saccharomyces cerevisiae. Nucleic Acids Res 23, 230-237. 
Sikorski, R. S. \& Hieter, P. (1989). A system of shuttle vectors and yeast host strains designed for efficient manipulation of DNA in Saccharomyces cerevisiae. Genetics 122, 19-27.

Strahl, T. \& Thorner, J. (2007). Synthesis and function of membrane phosphoinositides in budding yeast, Saccharomyces cerevisiae. Biochim Biophys Acta 1771, 353-404.

Styles, C. (2002). How to set up a yeast laboratory. Methods Enzymol 350, 42-71.
Tsong, A. E., Miller, M. G., Raisner, R. M. \& Johnson, A. D. (2003). Evolution of a combinatorial transcriptional circuit: a case study in yeasts. Cell 115, 389-399.

Yamagami, S., Morioka, D., Fukuda, R. \& Ohta, A. (2004). A basic helix-loop-helix transcription factor essential for cytochrome p450 induction in response to alkanes in yeast Yarrowia lipolytica. J Biol Chem 279, 22183-22189.

Edited by: V. J. Cid 